\title{
Bioanalysis
}

\section{7th British Mass Spectrometry Society annual meeting}

The 37th British Mass Spectrometry Society (BMSS) annual meeting took place over a brilliantly sunny 3 days by the sea in the historic Eastbourne Winter Gardens on the south coast of England. It was held between 13 and 15 September 2016. Twohundred attendees enjoyed a conference covering all aspects of MS with speakers drawn from across Europe and North America. The BMSS is particularly proud of the encouragement it offers students and early career scientists, both financially in the form of travel grants and also in terms of opportunities to present at an international level in a supportive atmosphere. Further encouragement to newcomers to the field is offered in the form of the Barber Prize for best oral presentation and the Bordoli Prize for the best poster. This year's winners were Patrick Knight (University of Leeds), the Bordoli Prize for the poster 'Characterising the Interaction of Ataxin-3 and the PolyGlutamine Aggregation Inhibitor QBP1'; Lisa Deininger (Sheffield Hallam University), the Barber Prize for 'Out Damned Spot! Bottom Up Proteomics for the Analysis of Bloodied Fingermarks'. In addition, the Delegates Choice for best poster went to Hannah Britt (Durham University) for 'Monitoring Reactions of Small Molecules with Cell Membranes by Liquid Chromatography-Mass Spectrometry'. For services to the MS community, Professor Gareth Brenton (Swansea University) was awarded the BMSS Medal; Professor Alison Ashcroft (Leeds University) and Anna Upton (former BMSS administrator) were given lifetime membership of the BMSS.

First draft submitted: 8 December 2016; Accepted for publication: 4 January 2017; Published online: 8 February 2017

Keywords: BMSS • conference • mass spectrometry

There were conference sessions on glycomics, protein complexes, MS solutions for challenging analytes, fundamentals/instrument developments, new MS technologies in proteomics, small molecules, emerging technologies for processing MS data, MS in environmental and forensic analysis, MS in translational medicine, MS in synthetic biology. The conference was preceded by an 'Introduction to Mass Spectrometry' training course, attended by 21 delegates.

Ranato Zenobi (Maccoll Lecturer, Zurich, Switzerland) gave an excellent talk titled 'Exhalomics by Ambient Mass Spectrometry', which gave an overview of the use of
Secondary ESI-MS (SESI-MS) in his laboratories for the analysis of breath (and other applications). In SESI, volatile compounds become ionized when they pass through an electrosprayed vapor. Analysis by MS allows real-time detection of analytes down to low parts-per-trillion by volume. When combined with accurate MS, SESI is a powerful tool for the identification of components in exhaled breath. The last $10 \mathrm{~s}$ of any exhalation is of particular interest as it represents the content of the alveolar space with less dilution by laboratory air. Examples given for the application of this technique included the monitoring of valproic acid, an antiepileptic drug, in breath
Patricia Wright $*, 1$

\& Christine Eckers ${ }^{2}$

'Smithers Rapra, Shawbury, Shropshire, SY4 4NR, UK

2Previously: Chair on British Mass Spectrometry Society Advisory Board *Author for correspondence: pwright@smithers.com 
to ensure clinical compliance; looking for diurnal patterns in breath components, potentially to tailor dosing to an individual's body clock; looking for novel biomarkers of chronic obstructive pulmonary disease (COPD); monitoring the pharmacokinetics of ketamine in mice, the real-time nature of the technique allows sampling every $10 \mathrm{~s}$; and monitoring changes in metabolites on the breath during obstructive sleep apnea. Professor Zenobi raised the possibility of establishing breath 'fingerprints' for individuals which reflects their phenotype, with multiple potential clinical applications [1].

Alison E Ashcroft (Robinson Lecturer, University of Leeds, UK) started her lecture on 'Insights into Protein Folding and Function: Are We Getting There?' with a review of the seminal work in the field of MS of native proteins. This include Professor Carol Robinson's work on ribosome and ATPase complexes [2,3], Katta and Chait's [4] studies on heme-globin complex in native myoglobin and, of course, John Fenn's [5] pioneering work on electrospray of large molecules. Professor Ashcroft then went on to describe the work within her own laboratories. Amyloid disease is caused by abnormal protein (amyloids) in tissues and organs. Amyloids are proteins which have folded abnormally, converting from their soluble, monomeric form into ordered, insoluble aggregates called amyloid fibrils. Alison Ashcroft identified the different conformers of $\beta 2$-microglobulin, which can accumulate in joints, using the Field Asymmetric Ion Mobility System (FAIMS; ThermoScientific, CA, USA) and Synapt (Waters, Wilmslow, UK) technology. Fifty small molecules were studied for their effect on fibril formation. It was found that the antibiotic rifampicin did inhibit fibril formation. It has been observed to bind to the folded protein enabling the dissociation of preformed fibrils [6].

Membrane proteins require a chaperone protein to assist the movement of the hydrophilic regions across the periplasm. The chaperone protein Skp assists the outer membrane proteins (OMPs) in their incorporation into membranes by both preventing folding of the protein and also enclosing protein in hydrophobic environment. Professor Ashcroft's group has studied the interaction between Escherichia coli Skp and various OMPs. They found that the Skp/OMP ratio required to prevent the folding depends on the size of the OMP. Small membrane protein may sit inside the Skp cavity whereas large membrane proteins may be coordinated to multiple copies of Skp [7].

\section{BMSS Chair's lecture Jack Henion, Cornell} University, Advion, Q2 Solutions, NY, USA What will MS look like in the future?

The conference ended with an entertaining lecture from Jack Henion. He started with a review of where MS has come in the last 20-30 years. The field of combining LC with MS, although it is now a routine analytical technique, was the cause of many headaches in the past. He highlighted some of the game-changers in MS such as the routine use of ESI, a great improvement to previous ionization techniques for LC-MS but not without its limitations. Other highlights included the development of very small MS detectors for chromatography and the development of the Orbitrap ${ }^{\mathrm{TM}}$.

In looking to the future, Jack reminded us to think big and challenge the limitations of the techniques and instrumentation we use. He highlighted areas where he could see MS will be increasingly going into the hands of the nonexperts and improvements in automation especially for data processing. He also more speculatively highlighted areas where he would like to see MS going in the future. In particular he would like to see more efficient use of mass spectrometers, for example, in the field of contract research, where there can be laboratories containing 20 or more LC-MS/MS instruments, all carrying out essentially the same experiments in parallel. He would like to see developments in the instrumentation to allow more channels of analysis on a single instrument, a definite challenge to the engineers. While electrospray has enabled analysis from small molecules to large protein complexes, the challenge of ion suppression and how this can affect MS, particularly for quantitative work, was highlighted and of all his wishes he would like to see a new ionization technique as an improvement to electrospray.

\section{Other highlights}

Andy Ray (AstraZeneca, Macclesfield, UK) described a novel use of selected ion flow tube-MS (SIFTMS) [8] for headspace analysis within the pharmaceutical industry. An agitator and GC injection port were combined with SIFT-MS to perform multiple headspace analysis of solid samples (first order kinetics were assumed). This system was successfully used to measure formaldehyde in the headspace of excipients. It also helped to identify methyl dioxolane as the smell generated in certain capsules (later confirmed by GC-MS).

Kevin Mills (Institute of Child Health, London, UK) introduced himself as a biochemist and lecturer in translational medicine at Great Ormond Street Hospital and head of the Biological Mass Spectrometry Research Centre at University College London. There are four main areas of clinical interest for his teams; anabolic defects, catabolic defects, biomarkers and new clinical tests. As example of the application of MS in a clinical situation, Dr Mills discussed Fabry Disease. This is a rare but painful condition arising from deficiency of the enzyme $\alpha$-galactosidase. The original lab- 
oratory test for Fabry disease was an enzyme assay but Kevin Mills' laboratory has developed a mass spectral assay for the measurement of globotriaosylceramide as a biomarker for diagnosis and monitoring [9]. An area of particular clinical need for rapid and accurate diagnosis is for neurodegenerative disorders. It is predicted that a third of people over 65 will die of dementia. Lewy Body dementia is a form of Alzheimer's disease. The current method of diagnosis requires immunoassay of diagnostic peptides in CSF. Dr Mills' group has developed a 2D LC-MS/MS method to measure the biomarkers of dementia without the requirement for a label [10].

Perdita Barran (University of Manchester) started her presentation by reminding the audience about key work in the field:

- Kemper and Bowers [11] showed that $\mathrm{Co}^{+}$has two electronic states which can be separated via their differing ion mobilities;

- Martin Jarrold's group [12] investigated silicon clusters. They used trajectory calculations to determine geometries and found that these values were in good agreement with experimentally determined values;

- Warnke et al. [13] showed that ion mobility spectrometry (IMS) could be used to separate the $\mathrm{N}$ - and $\mathrm{O}$ - protomers of benzocaine.

Professor Barran's group is particularly known for its IM-MS work on large molecules, but they have also been involved in some interesting studies of smaller molecules. In conjunction with the University of Edinburgh, they have looked at the separation of the different conformations of molecular trefoil knots. The knotted and extended conformations could be distinguished on the basis of their collision cross section (CCS) [14]. She also discussed the application of IM-MS to determine the nature of the glycosidic linkage $(\alpha$ or $\beta$ ) to carbohydrates.

Caroline Dessent (University of York, York, UK) adapted a commercial MS/MS mass spectrometer to incorporate a laser between the first and second mass analysers, to perform UV photodissociation (of molecules containing a UV chromophore) rather than collision-induced dissocation (CID). The technique allowed discrimination between protomers and deprotomers, that is molecules protonated and deprotonated at different sites [15].

Kayleigh Arthur (Loughborough University, Loughborough, UK) used a miniaturized FAIMS, the UltraFAIMS by Owlstone (Cambridge, UK), with mass spectrometric detection to do targeted and untargeted metabolite identification in human urine. Ion mobility separations were achievable within the timescale of elution of each of the chromatographic peaks, that is, there were ion mobility separations underneath the chromatographic separations. In addition to teasing out isomers, the use of FIAMS increased the $\mathrm{S} / \mathrm{N}$ by nearly 50 -fold. The UltraFAIMS was also compatible with $\mu$ PLC.

Florence Lai (Syngenta Ltd., Bracknell, UK) presented on the overcoming the challenge of measuring the insecticide DDT (1,1'-(2,2,2-trichloroethane1,1-diyl)bis(4-chlorobenzene)) in a total of just five mosquitoes. The current analytical methods usually have the luxury of more starting material, so Florence had to be creative. Thermal desorption GCMS was tried but was found that these low levels of DDT were masked by extractables from the containers used for sample preparation. The answer was GC/GC-MS for the removal of interfering material. This method was successfully applied to measure levels down to $2 \mu \mathrm{g}$.

Robert Mistrik (HighChem, Slovakia) described his company's database, mzCloud [16]. This database contains a massive amount of high-quality data. It contains over a 1.5 million mass spectra derived from over 5500 compounds, collected at a range of collision energies. The spectra are supplied by both academic and industrial partners. Spectra are checked for calibration (about $20 \%$ of data are from poorly calibrated instruments) and are recalibrated if necessary. Approximately $10 \%$ of records have quantum chemistry calculations associated, which can be used as an aid to spectral interpretation. They are also filtered to remove interferences. Breakdown curves (a plot of remaining parent ion relative to the total product ions at different collision energies) are generated when possible as they can be characteristics of the compound. mzCloud also contains spectral trees and is searchable for both structures and substructures. mz Cloud appeared to be a thorough, well-thought through and continually expanding database.

\section{Summary}

This was a vibrant meeting with a high level of engagement from participants. The feedback from attendees was that the excellent quality of the both the talks and posters, together with the variety of topics covered, made for a stimulating experience, as attested to by the conversations at the social events. Also, we were fortunate to have blue skies which showed this seaside venue to its best.

Planning for the 38th BMSS annual meeting 2017 is underway. This will be held at the Royal Northern College of Music in Manchester (5-7th September 2017), preceded by an LC-MS training course. 
This central venue provides a stunningly contemporary setting for presentation of cutting-edge science. The conference dinner will be held at the Museum of Science and Industry. The Chair's invited speaker has been confirmed as Graham Cooks (Purdue University, IN, USA) and the Maccoll Lecturer will be Lars Konermann (University of Western Ontario, ON, Canada). For further details, see the BMSS website [17].

\section{References}

1 Martinez-Lozano Sinues P, Zenobi R, Kohler M. Analysis of the exhalome. Chest 144(3), 746-749 (2013).

2 McKay AR, Ruotolo BT, Ilag LL, Robinson CV. Mass measurements of increased accuracy resolve heterogeneous populations of intact ribosomes. J. Am. Chem. Soc. 128(35), 11433-11442 (2006).

3 Schmidt C, Zhou M, Marriott H, Morgner N, Politis A, Robinson CV. Comparative cross-linking and mass spectrometry of an intact F-type ATPase suggest a role for phosphorylation. Nat. Commun. 4, 1985 (2013).

4 Katta V, Chait BT. Observation of the heme-globin complex in native myoglobin by electrospray-ionization mass spectrometry. J. Am. Chem. Soc. 113(22), 8534-8535 (1991).

5 Fenn JB. Electrospray wings for molecular elephants (Nobel lecture). Angew. Chem. Int. Ed. Engl. 42(33), 3871-3894 (2003).

6 Leney A, Pashley C, Scarff C, Radford S, Ashcroft A. Insights into the role of the beta-2 microglobulin D-strand in amyloid propensity revealed by mass spectrometry. Molecular BioSystems 10 (3), 412-420 (2014).

7 Schiffrin B, Calabrese AN, Devine PWA et al. Skp is a multivalent chaperone of outer-membrane proteins. Nat. Struct. Mol. Biol. 23(9), 786-793 (2016).

8 Spanĕl P, Smith D. Progress in SIFT-MS: breath analysis and other applications. Mass Spectrom. Rev. 30(2), 236-267 (2011).

9 Boscaro F, Pieraccini G, la Marca G et al. Rapid quantitation of globotriaosylceramide in human plasma and urine: a

\section{Financial \& competing interests disclosure}

The authors have no relevant affiliations or financial involvement with any organization or entity with a financial interest in or financial conflict with the subject matter or materials discussed in the manuscript. This includes employment, consultancies, honoraria, stock ownership or options, expert testimony, grants or patents received or pending, or royalties.

No writing assistance was utilized in the production of this manuscript.

potential application for monitoring enzyme replacement therapy in Anderson-Fabry disease. Rapid Commun. Mass Spectrom. 16(16), 1507-1514 (2002).

10 Mattsson N, Zegers I, Andreasson U et al. Reference measurement procedures for Alzheimer's disease cerebrospinal fluid biomarkers: definitions and approaches with focus on amyloid $\beta 42$. Biomark. Med. 6(4), 409-417 (2012).

11 Radi PP, Hsu M-T, Rincon ME, Kemper PR, Bowers MT. On the structure, reactivity and relative stability of the large carbon cluster ions $\mathrm{C}+62, \mathrm{C}+60$ and $\mathrm{C}+58$. Chem. Phys. Lett. 174(3-4). 223-229 (1990).

12 Ho K-M, Shvartsburg AA, Pan B et al. Structures of medium-sized silicon clusters. Nature 392 (6676), 582-585 (1998).

13 Warnke S, Seo J, Boschmans J et al. Protomers of benzocaine: solvent and permittivity dependence. J. Am. Chem. Soc. 137(12), 4236-4242 (2015).

14 Barran PE, Cole HL, Goldup SM et al. Active-metal template synthesis of a molecular trefoil knot. Angew. Chem. Int. Ed. Engl. 50(51), 12280-12284 (2011).

15 Matthews E, Dessent CEH. Locating the proton in nicotinamide protomers via low-resolution UV action spectroscopy of electrosprayed solutions. J. Phys. Chem. 120(46), 9209-9216 (2016).

16 mzCloud - Advanced Mass Spectral Database. www.mzcloud.org/

17 British Mass Spectrometry Society. www.bmss.org.uk 